\title{
Desenvolvimento de Blendas Poliméricas visando a Tenacificação dos Polímeros: Uma revisão
}

\section{Development of Polymer Blends in order to Toughening of Polymers: A review}

\author{
Carlos Bruno Barreto Luna ${ }^{1}$; Divânia Ferreira da Silva²; Sabrina Kelly Trajano \\ Basílio $^{3}$; Edcleide Maria Araújo ${ }^{4}$; Adriano Lima da Silva ${ }^{5}$; Ana Flávia Camara \\ Bezerra $^{6}$
}

\begin{abstract}
Resumo
Polímeros são materiais de grande utilização nos diversos setores da economia mundial. A utilização de materiais poliméricos no cotidiano, em substituição aos materiais clássicos vem crescendo nas últimas décadas. Porém, para determinadas aplicações estruturais os polímeros precisam ser tenacificados. Uma das principais técnicas de tenacificação se baseia na mistura física de dois ou mais componentes, formando as chamadas blendas poliméricas. A adição de borrachas ou desvulcanizados em composições poliméricas é apresentada na literatura como uma maneira de gerar misturas de fácil processamento, vantajosas economicamente e com aumento da tenacidade da matriz termoplástica de interesse. Além disso, pode ser alternativa para o reaproveitamento dos resíduos de pneus e calçados advindos de indústrias, bem como reduzir os efeitos nocivos sobre o meio ambiente. Portanto, o presente trabalho tem como objetivo apresentar uma revisão sobre as definições, vantagens, fundamentos termodinâmicos e tenacificação de polímeros.
\end{abstract}

Paravras chave: Blendas poliméricas. Tenacificação. Borracha.

\begin{abstract}
Polymers are materials of large use in the various sectors of the world economy. The use of polymeric materials in daily life, instead of the classic materials has increased in recent decades. However, for certain structural applications polymers need to get tougher. One of the principal toughening techniques based on physical mixture of two or more components, forming the so-called polymer blends. The addition of rubber or not vulcanized in polymer compositions is reported in the literature as a means of generating mixtures of easy processing, and economically advantageous to increase the toughness of the thermoplastic matrix of interest. Moreover, it can be an alternative for the recycling of waste tires and footwear coming from industries, as well reduce harmful effects on the environment. Therefore, the present study aims to present a review of the definitions, benefits, thermodynamic fundamentals and toughening polymers.
\end{abstract}

Keywords: Polymer blends. Toughening. Rubber.

\footnotetext{
${ }^{1}$ Aluno PIBIC Engenharia de Materiais, Universidade Federal de Campina Grande; brunobarretodemaufcg@hotmail.com

${ }^{2}$ Doutoranda em Ciência e Engenharia de Materiais pela Universidade Federal de Campina Grande; divaniaf@yahoo.com.br

${ }^{3}$ Aluna PIVIC Engenharia de Materiais, Universidade Federal de Campina Grande; sabrina_materiais@hotmail.com

${ }^{4}$ Doutora em Engenharia de Materiais pela Universidade Federal de São Carlos. Professora Ássociada; edcleide@dema.ufcg.edu.br.

${ }^{5}$ Aluno PIBIC Engenharia de Materiais, Universidade Federal de Campina Grande; adrianolimadasilva@hotmail.com

${ }^{6}$ Doutoranda em Ciência e Engenharia de Materiais pela Universidade Federal de Campina Grande; anaflavia.dema@yahoo.com.br
} 


\section{Introdução}

Atualmente a proteção do meio ambiente tornouse um sinal de qualidade de vida. A qualidade do meio ambiente passa, particularmente, pela recuperação dos produtos usados, sua reutilização e reciclagem, antes deles começarem a poluir o ambiente (LUNA; SILVA; ARAÚJO, 2014). Os resíduos industriais vêm se tornando um dos mais sérios problemas que a sociedade enfrenta. Sua disposição de forma inadequada provoca a degradação do meio ambiente, bem como sérios transtornos sociais. A elaboração de um modelo autossustentável, na questão ambiental, desponta como objetivo principal dentro de uma sociedade consciente. $\mathrm{O}$ reaproveitamento desses resíduos industriais na cadeia produtiva surge como alternativa para minimizar danos e tem dado certo em vários países do mundo (COLLATTO; BERGMANN; RAMIRES, 2008).

A demanda de artefatos poliméricos vem crescendo consideravelmente, desde as décadas passadas até os dias atuais. Os polímeros vêm substituindo muitos materiais caros e mais pesados, logo a sua percentagemnos lixões tem um crescimento contínuo. Isto pode ser atribuído ao baixo custo, excelente versatilidade de aplicações e desempenho. No entanto, como os materiais poliméricos não se decompõem facilmente, a deposição destes resíduos constitui um sério problema ambiental (BOM, 2009; ARAÚJO, 2011).

Grande importância tem sido dada à reciclagem de materiais plásticos. Programas governamentais visando promover a conscientização da população para os problemas associados ao descarte do lixo urbano e industrial fizeram com que, hoje, as reciclagens de metais, vidros e materiais poliméricos sejam realizadas em diversas partes do mundo (ARAÚJO; CARVALHO; FOOK, 1997). A utilização destes materiais reciclados traz como vantagens a redução de deposições irregulares e como consequência minimiza o descarte de rejeitos próximo das zonas urbanas (CARMO; MAIA; CÉSAR, 2012). Além disso, o reaproveitamento destes rejeitos proporciona vantagens econômicas quando se trata de processos industriais, além de contribuir na redução do impacto ambiental decorrente da disposição inadequada dos resíduos industriais e na preservação dos recursos naturais. Os aterros sanitários encontram-se a beira de um colapso, devido a não acompanharem o rápido crescimento do volume de lixo (AMÂNCIO; MEGOD, 2011). Na Tabela 1 estão apresentados os números referentes ao volume de produção total de pneus das indústrias no Brasil.

Tabela 1 - Volume de Produção de Pneus de 2006 a 2013

\begin{tabular}{|c|c|}
\hline Produção Brasileira (Milhares de Unidades) \\
\hline Ano & Total \\
\hline $\mathbf{2 0 0 6}$ & $54.467,00$ \\
\hline $\mathbf{2 0 0 7}$ & $57.247,70$ \\
\hline $\mathbf{2 0 0 8}$ & $57.247,70$ \\
\hline $\mathbf{2 0 0 9}$ & $54.085,90$ \\
\hline $\mathbf{2 0 1 0}$ & $67.305,10$ \\
\hline $\mathbf{2 0 1 1}$ & $66.926,60$ \\
\hline $\mathbf{2 0 1 2}$ & $62.661,00$ \\
\hline
\end{tabular}

Fonte: (ANIP, 2014)

Cada uma das partes do pneu é composta por diferentes materiais que garantem o seu funcionamento. Os componentes utilizados para a fabricação da borracha necessária à produção de pneus têm uma mistura de borracha sintética, borracha natural, óleos, enxofre, negro de fumo, óxido de zinco, entre outros componentes químicos. O elastômero mais utilizado na confecção de pneus é o copolímero estireno-butadieno (SBR), contendo $25 \%$, em peso, de estireno (GOMES FILHO, 2007).

Os resíduos de borracha oriundos das indústrias de calçados e de pneus são fonte de poluição ambiental e desperdício de matéria-prima com boas propriedades e alto valor agregado. Sabese que estes materiais apresentam resistência relativamente alta a agentes biológicos e às intempéries, causando assim sérios problemas à comunidade em geral quando descartados. Tanto os 
aspectos econômicos quanto a poluição ambiental são justificativas para que se envidem esforços no sentido de promover a reciclagem destes materiais poliméricos (SILVA; ARAÚJO; MELO, 2012). O reaproveitamento das borrachas é dificultado pelo fato destes materiais possuírem composição muito complexa e heterogênea bem como apresentarem estrutura reticulada, o que torna o material infusível, dificultando ou até mesmo impedindo o seu reprocessamento (SCHUSTER, 1993). A mistura de polímeros ou blendas poliméricas é uma das estratégias para o reaproveitamento dos resíduos de borrachas.

A substituição da reciclagem de polímeros puros por misturas de polímeros descartados é economicamente interessante, pois a reciclagem provoca a quebra de cadeias do polímero puro prejudicando suas propriedades. A blenda de dois ou mais polímeros descartáveis pode resultar num material de baixíssimo custo e com propriedades interessantes, sendo também uma alternativa inteligente para o reaproveitamento da borracha (WIEBECK; HARADA, 2005).

\section{Blendas Poliméricas}

Blendas poliméricas são sistemas originários da mistura física de dois ou mais polímeros e/ou copolímeros com o objetivo de obter propriedades desejadas. Para ser considerada uma blenda, os compostos devem ter concentração acima de $2 \%$ em massa do segundo componente (PASSADOR; PESSAN; RODOLFO JR, 2006). As blendas constituem alternativa para a obtenção de materiais poliméricos com propriedades que, em geral, não são encontradas em um único material (ARAÚJO; HAGE JR; CARVALHO, 2003). O interesse no estudo das blendas tem aumentado a cada ano. Os principais motivos para esse crescimento se dá pelas vantagens:

- Tecnológica: as blendas possuem a capacidade de combinar as propriedades de diferentes componentes de modo a obter excelentes propriedades mecânicas, térmicas e químicas.
- Financeira: o custo de desenvolvimento de uma blenda é menor que o custo da síntese de um novo polímero.

- Ambiental: Possibilidade de reciclagem industrial.

- Mercadológico: Possibilidade de ajuste da composição da blenda de acordo com as especificações do cliente (FERNANDES et al., 2012).

O interesse por misturas poliméricas tem crescido continuamente durante as últimas décadas. Os setores que mais estão crescendo nessa área são: o automotivo, elétrico e eletrônico, de embalagem, de construção, aeronáutica, e de utensílios doméstico (KONING et al., 1998). A mistura de polímeros constitui alternativa economicamente viável para obter materiais com características físicas, químicas e físico-químicas melhores do que aquelas dos polímeros puros. Os componentes da blenda são selecionados de modo a conservar as vantagens de cada polímero. A grande atenção dada às blendas, tanto do ponto de vista acadêmico, quanto industrial/ comercial, é devido à relativa facilidade na obtenção de materiais com propriedades desejadas, sem altos investimentos aplicados no desenvolvimento da síntese de novos polímeros. Dessa maneira, as blendas são uma versátil solução tecnológica para se obter materiais poliméricos com as mais diversas especificações a um custo relativamente baixo por meio de combinações de polímeros com as propriedades de interesse (SILVA, 2011).

Muitos polímeros amorfos ou vítreos tendem a apresen $\neg$ tar comportamento frágil, limitando assim sua gama de aplicações. E uma das maneiras de solucionar esse problema é a tenacificação por meio uso de elastômeros como modificadores de impacto para esses materiais com a produção de blendas poliméricas. A mistura com elastômeros é reconhecidamente a técnica mais utilizada por grande parte das in $\neg$ dústrias de polímeros para aumentar a resistência ao impacto e a tenacidade desses materiais (ROVERE et al., 2008). Várias 
são as maneiras de obtenção de blendas e uma classificação destas pode ser feita por meio dos métodos de obtenção, que são: por solução, por reticulados poliméricos interpenetrantes (IPN) e por mistura mecânica no estado fundido.

O processo de obtenção de blenda por solução envolve a dissolução dos polímeros em um solvente comum, com posterior evaporação do solvente e a obtenção de um filme (MANO; MENDES, 2001). $\mathrm{O}$ aquecimento pode ser utilizado para aumentar o grau de solubilidade dos componentes individuais ou da mistura. A etapa mais importante é a evaporação do solvente, que normalmente é feita por meio da formação de um filme e posterior evaporação à temperatura ambiente, em estufa ou sob vácuo. Esse tipo de blenda é de baixa produtividade e geralmente são desenvolvidas em laboratório.

As blendas por reticulado polimérico interpenetrantes (IPN) são obtidas por uma mistura polimérica, onde os constituintes estão na forma de reticulados que se interpenetram e formam um único reticulado, sem que haja qualquer tipo de reação química entre eles. Quando somente um dos constituintes está na forma reticulada estes tipos de blendas são conhecidos como semi-IPNs. Os IPNs são tipos especiais de blendas poliméricas e são utilizados para melhorar a interação entre fases e a compatibilidade de blendas por solução e por mistura mecânica (SILVA, 2011).

As blendas obtidas por mistura mecânica podem ser processadas por meio de extrusão, injeção ou misturadores intermitentes. A mistura mecânica envolve aquecimento e alto cisalhamento e é o método mais utilizado industrialmente por razões econômicas e porque permite a mistura de polímeros em grande escala (HAGE JR; PESSAN, 2001).

\section{Miscibilidade de Blendas Poliméricas}

A mistura de diferentes tipos de polímeros frequentemente produz blendas poliméricas miscíveis, imiscíveis ou parcialmente miscíveis A miscibilidade relaciona-se com a capacidade de dois ou mais componentes se misturarem em nível molecular, resultando numa mistura homogênea. Uma forma simples de verificação da miscibilidade pode ser feita através do número e posição das Tg's do material. A Tg de um polímero é a temperatura na qual a amostra passa de um estado vítreo para uma estrutura em que as cadeias têm maior mobilidade (MOTHE; AZEVEDO, 2002).

A maior parte das blendas poliméricas é imiscível devido a razões termodinâmicas, podendo ainda apresentar incompatibilidade, resultante da baixa dispersão de uma das fases na matriz e da baixa adesão entre estas. Uma boa dispersão e adesão das fases podem ser alcançadas pela adição de um compatibilizante adequado, em geral um copolímero em bloco ou de enxertia, que atua na interface, reduzindo a tensão interfacial. Este copolímero pode, em princípio, ser obtido separadamente e, então, adicionado à mistura polimérica (GAN; PAUL, 1994).

Nas blendas miscíveis, os polímeros formam uma única fase e estão intimamente misturados em nível molecular, apresentando somente um valor de $\mathrm{Tg}$, entre os valores das $\mathrm{Tg}$ 's dos polímeros individuais. Nesse tipo de blenda, os polímeros estão dispersos ao acaso e interações intermoleculares favoráveis provavelmente ocorrem entre os dois componentes da blenda. Já os componentes das blendas imiscíveis são essencialmente independentes. Este tipo de blenda apresenta um número de fases relacionado ao número de seus componentes. Consequentemente, se os componentes individuais da blenda imiscível apresentar transições vítreas, espera-se que as blendas apresentem valores de $\mathrm{Tg}$ praticamente iguais às dos polímeros individuais. As blendas parcialmente miscíveis devem apresentar, então, valores de $\mathrm{Tg}$ relativos aos seus componentes, mas situados entre os valores dos polímeros individuais (SPERLING, 2001). 
O estudo e a obtenção de blendas são, assim, uma saída para a modificação das propriedades de materiais poliméricos. As blendas de polímeros miscíveis são materiais que podem apresentar propriedades mecânicas melhores que as de cada um dos polímeros isolados. Entretanto, as blendas com miscibilidade muito baixa apresentam separação de fases, e neste caso, as suas propriedades vão depender das propriedades de cada fase (SILVA, 2011).

O critério que controla os aspectos de miscibilidade de sistema polimérico está baseado na equação de energia livre de mistura, também conhecido como equação de energia livre de Gibbs $\left(\Delta \mathrm{G}_{\mathrm{m}}\right)$, que é dada por:

$$
\Delta G_{m}=\Delta H_{m}-T \Delta S_{m}
$$

Em que: T é a temperatura absoluta, $\Delta \mathrm{H}_{\mathrm{m}}$ e $\Delta \mathrm{S}_{\mathrm{m}}$ são a variação da entalpia e da entropia de mistura, respectivamente. $\Delta \mathrm{G}_{\mathrm{m}}$ é afetada pela composição e pela temperatura do sistema. Assim, para que a miscibilidade ocorra, $\Delta \mathrm{Gm}$ deve ser menor que zero $\left(\Delta \mathrm{G}_{\mathrm{m}}<0\right)$ e satisfazer a condição abaixo, a qual assegura a estabilidade contra a segregação de fases:

$$
\left(\frac{\partial^{2} \Delta G_{m}}{\partial \phi_{i}^{2}}\right)_{T, p}>0
$$

em que: $\Phi_{i}$ é a fração em volume de cada componente, é a notação da derivada parcial, T é a temperatura e p é a pressão.

A termodinâmica estatística possibilita o cálculo das contribuições da entalpia e da entropia de mistura para a energia livre de Gibbs $\left(\Delta \mathrm{G}_{\mathrm{m}}\right)$, utilizando um modelo de rede tridimensional, no qual cada sítio é ocupado por um segmento de cadeia do polímero. $\mathrm{O}$ fato de a maioria dos pares poliméricos não formar sistemas miscíveis deve-se à pequena variação de entropia $\left(\Delta \mathrm{S}_{\mathrm{m}}\right)$ combinatorial resultante da mistura de dois polímeros de alta massa molar. Essa entropia pode ser representada pela equação (3), na qual $\mathrm{R}$ é a constante dos gases; $\mathrm{V}$ o volume da blenda, VR um volume de referência (usualmente o volume molar da menor unidade repetitiva); $\varphi_{1}$ e $\varphi_{2}$ as frações volumétricas dos polímeros 1 e $2 ; \mathrm{X}_{1}$ e $\mathrm{X}_{2}$ são os graus de polimerização dos polímeros 1 e 2 .

$$
\Delta S_{m}=\left(\frac{R V}{V_{R}}\right)\left[\left(\frac{\Phi_{1}}{X_{1}}\right) \operatorname{In} \Phi_{1}+\left(\frac{\Phi_{2}}{X_{2}}\right) \operatorname{In} \Phi_{2}\right]
$$

A entalpia de mistura pode ser expressa como um parâmetro de interação adimensional por segmentos de polímero, $\mathrm{X}_{\mathrm{ij}}$, baseado na fórmula de Van Laar:

$$
\Delta H_{m}=R T\left(\frac{V}{V_{R}}\right) X_{i j} \Phi_{i} \Phi_{j}
$$

Assim, a equação da energia livre $\left(\Delta \mathrm{G}_{\mathrm{m}}\right)$ pode ser escrita sob a forma (5):

$$
\Delta G_{m}=\left(\frac{R T V}{V_{R}}\right)\left[\left(\frac{\Phi_{1}}{X_{1}}\right) \operatorname{In} \Phi_{1}+\left(\frac{\Phi_{2}}{X_{2}}\right) \operatorname{In} \Phi_{2}+X_{12} \Phi_{1} \Phi_{2}\right]
$$

em que $X_{12}$ é o parâmetro de interação de FloryHuggins correspondente à mistura dos polímeros 1 e 2 (AKCELRUD, 2007).

Levando em consideração que os graus de polimerização $X_{1}$ e $X_{2}$ que aparecem no denominador da equação (3) são grandes, o valor de $\Delta \mathrm{S}_{\mathrm{m}}$ será pequeno e, portanto, para $\Delta \mathrm{G}_{\mathrm{m}}<0$, é preciso que $\Delta \mathrm{H}_{\mathrm{m}} \leq 0$ ou tenha um valor positivo muito pequeno. Isso implica na necessidade de interações intermoleculares favoráveis entre os componentes, tais como forças dipolares, ligações hidrogênio, etc. A equação também mostra que aumento na massa molar direciona o sistema para maior imiscibilidade. Isso acontece porque, quanto maior o número de pontas, maiores são 
as possibilidades conformacionais, aumentando a entropia configuracional e favorecendo a miscibilidade (AKCELRUD, 2007).

\section{Compatibilidade de Blendas Poliméricas}

Miscibilidade e compatibilidade são termos muito confundidos na literatura. Como visto anteriormente, a miscibilidade é governada pelos aspectos termodinâmicos da mistura, ou seja, é uma característica intrínseca do sistema. Por outro lado, muitos sistemas poliméricos de interesse acadêmico e comercial são imiscíveis, ou seja, apresentam mais de uma fase. Assim, a interface nestes sistemas é de extrema importância no comportamento final do material. O melhor desempenho destas blendas pode ser conseguido pela redução da tensão interfacial e pelo aumento da dispersão e da adesão entre as fases, fatores estes, que propiciam a estabilização da morfologia. Assim, a compatibilidade é uma característica que pode ser obtida por meio da modificação do sistema para a obtenção das propriedades desejadas, ou seja, a compatibilidade é um aspecto tecnológico (SILVA, 2011).

Em geral, a compatibilização é feita pela introdução de um componente que atue como um "agente" interfacial entre as fases do sistema. Este componente, chamado de compatibilizante, pode ser adicionado ao sistema ou gerado in situ durante o processamento da blenda. Neste segundo método, conhecido como compatibilização reativa, mistura reativa ou processamento reativo, os componentes da blenda são passíveis de reações químicas no estado fundido, obtendo-se um copolímero que apresenta diferentes segmentos de cadeia, proveniente dos diferentes componentes da blenda. $\mathrm{O}$ agente compatibilizante gerado localiza-se preferencialmente na região interfacial, e seu papel é similar aos agentes emulsificantes de baixa massa molar utilizados em sistemas líquidos imiscíveis (CHEN; WHITE, 1993).

A interface em blendas poliméricas imiscíveis é caracterizada por um gradiente de concentração entre os domínios dos diferentes componentes. Assim, pode-se designar esta região interfacial como uma interfase, ou seja, uma terceira fase com características próprias que interferem drasticamente nas propriedades finais do sistema (UTRACKI, 1989). A espessura da interfase depende das interações entre os polímeros, da massa molar e da concentração das fases. De maneira geral, esta espessura está em torno de 2 e 50nm dependendo da afinidade entre as fases (STAMM; SCHUBERT, 1995).

A resistência da interface é dada pelas interações intermoleculares entre os componentes dos sistemas. A resistência adesiva das fases pode ser obtida por meio de ligações químicas ou interações intermoleculares. A energia de uma ligação química está em torno de $80 \mathrm{kcal}^{\mathrm{mol}}{ }^{-1}$, enquanto que a força das atrações de van der Waals encontram-se em torno de 2,5 kcal.mol ${ }^{-1}$. Assim, dependendo da resistência desejada, a interface pode ser alterada utilizando-se compatibilizantes com grupos funcionais passíveis de reações químicas, ou que apenas promovam a interação entre as fases (AJJI; UTRACKI, 1996).

\section{Tenacificação de Polímeros}

A tenacidade é a capacidade de absorção deformacional de impacto, isto é, absorção de energia por um determinado material até sua fratura e é um dos parâmetros mais importantes que determina se um dado polímero pode ser usado como material de engenharia. $\mathrm{O}$ grande desafio na produção de materiais tenacificados por elastômeros não é somente a melhora na resistência à fratura, mas também a aquisição desta propriedade com a mínima perda de outras (resistência à tração, módulo de elasticidade, viscosidade, transparência) a custos razoáveis. Nas misturas de termoplásticos reforçados com elastômeros, quando se aplica uma tensão, as partículas elastoméricas dispersas concentram ou 
absorvem essa tensão, provocando uma alteração do estado de tensão da fase matricial e uma intensa deformação plástica, melhorando assim a resistência ao impacto do material (COSTA, 2009).

É conveniente classificar os mecanismos de deformação em polímeros vítreos como escoamento por cisalhamento ("shear yielding") e escoamento por microfibrilamento ("crazing"). Dependendo das características do polímero e do estado de tensão aplicado, pode ocorrer um ou outro mecanismo, podendo também ocorrer os dois mecanismos simultaneamente, com ou sem interação um com o outro (OLIVEIRA, 2009).

No escoamento sob cisalhamento, a deformação consiste de uma distorção do formato do material sem mudança significativa no volume da amostra, ocorrendo desta maneira, uma mudança permanente no corpo de prova. As moléculas do polímero podem retornar à sua posição original, quando ele for submetido a um tratamento térmico acima da temperatura de transição vítrea, Tg. Em materiais cristalinos, ocorrem em planos de deslizamento específicos, os planos cristalinos. Já em materiais amorfos, ocorre por meio do movimento cooperativo das moléculas, e são, portanto, muito menos localizados. Quando ocorre escoamento bem localizado, são originados bandas de cisalhamento ("shear yielding). Estas bandas são iniciadas em regiões onde há pequenas heterogeneidades de tensão e um considerável grau de orientação molecular é observado nas mesmas (SILVA, 2011).

A formação do fenômeno microfibrilamento, um mecanismo alternativo de deformação nos polímeros, é um processo de escoamento localizado. Quando uma tensão de tração é aplicada a um polímero frágil, pequenos vazios formam-se num plano perpendicular ao da tensão aplicada, ocasionando o início de uma trinca. Dependendo da condição de tensão, os vazios não coalescem para formar uma trinca verdadeira, pois são estabilizados por fibrilas de polímero orientado, as quais unem as duas partes do vazio e permitem sustentar um alto nível de tensão. A região onde ocorrem tais fenômenos é denominada "craze" (OLIVEIRA, 2009).

A absorção de energia por este mecanismo de deformação ocorre por meio da energia dissipada pelo desemaranhamento e escoamento localizado de cadeias, formando fibrilas que impedem a coalescência prematura dos "crazes" e também ocorre pela criação de novas superfícies. Apesar do processo de "crazing" também promover deformação plástica, geralmente a dissipação de energia é bem menor do que no processo de "shear yielding", pois o escoamento é muito localizado. A fratura do polímero ocorre quando a tensão nas fibrilas se torna tão alta que estas se rompem, permitindo a propagação do microvazio formado que origina uma trinca macroscópica (OLIVEIRA, 2009).

O microfibrilamento inicia-se no equador das partículas discretas, e fibrilas de material polimérico orientado são desenvolvidas na direção da tensão aplicada, para estabilizar ou inibir os vazios, no plástico reforçado com borracha. A vantagem da presença das partículas de borracha é que elas devem estar distribuídas uniformemente por todo o material, estimulando com maior eficácia o desenvolvimento de concentração de tensão. $\mathrm{O}$ resultado é uma superfície escoada, tanto maior quanto for à quantidade de microfibrilamento, consistindo de um conjunto interpenetrante de vazios e fibrilas poliméricas mais estáveis entre as partículas de borracha. Todo esse processo absorve e dissipa bastante energia deformacional, aumentando assim a resistência ao impacto do material. Em linhas gerais, o microfibrilamento ocorre num plano perpendicular à tensão aplicada e inicia-se na interface borracha-matriz. Sob tensão consideravelmente alta, a estrutura fibrilar se rompe, formando a trinca que, eventualmente, pode se propagar quando atingir um tamanho crítico. Então, o microfibrilamento é o primeiro 
estágio da trinca e as partículas de borracha devem funcionar não apenas iniciando o mecanismo, mas também retardando a fratura (ARAÚJO, 2001; OLIVEIRA, 2009; NEWMAN; PAUL, 1978).

Em polímeros tenacificados com borracha, os mecanismos de tenacificação são os mesmos que os observados em polímeros vítreos, ou seja, microfibrilação e escoamento sob cisalhamento. O que determina a ocorrência de um ou de outro mecanismo é o comportamento de fratura da matriz polimérica (frágil ou dúctil). Polímeros frágeis requerem boa adesão das partículas de borracha à matriz e a microfibrilação é o mecanismo de tenacificação predominante. Em polímeros dúcteis, adesão não é um requisito crítico e o escoamento por cisalhamento é considerado o principal mecanismo de deformação. $O$ escoamento sob cisalhamento, de uma maneira geral, ocorre a partir da interface partícula-borracha, através do microescoamento por cisalhamento das moléculas da matriz, ou seja, acontece na matriz, pela microdeformação plástica no polímero. Por isso, a concentração de tensão originada pelas partículas de borracha é necessária para que sejam iniciadas as bandas de cisalhamento e as microtrincas. Nos polímeros semicristalinos como a poliamida 6, esse fenômeno foi observado por meio do microescoamento das moléculas que formam as bandas de cisalhamento. Nos polímeros amorfos, embora com menor frequência, por meio de bandas de cisalhamento ou zonas de cisalhamento difusas. Em copolímeros de ABS, materiais com mais de uma fase, esse fenômeno foi observado pelas propriedades mecânicas e pela técnica de microscopia ótica. Percebeu-se que, quando submetidos a testes sob tração, havia uma distorção das partículas de borracha, pois a interface partícula-matriz concentra tensão, ou seja, havia um campo de tensão e, ao redor de quase todas as partículas, havia deformação plástica localizada, como resultado do escoamento das moléculas do polímero (ARAÚJO, 2001; HAGE JR; PESSAN, 2001; OLIVEIRA, 2009).

\section{Revisão Bibliográfica Específica}

Araújo, Carvalho e Fook (1997) adicionaram resíduos de borracha, provenientes de indústrias de calçados e de pneus ao poliestireno visando tenacificá-lo. Os efeitos da concentração (5-25\% em peso) e granulometria (1200 a $180 \mathrm{~mm}$ ) dos resíduos nas propriedades mecânicas destas blendas foram avaliados e as suas superfícies de fratura examinadas. Ficou evidenciado que a resistência à tração tende a diminuir e a resistência ao impacto aumentar conforme o teor de borracha aumenta e a granulometria diminui. A análise morfológica das superfícies de fratura confirmaram estes resultados. As amostras obtidas pelo processo de injeção apresentaram maior uniformidade e, consequentemente, melhores propriedades mecânicas.

Martínez-Barrera, López e Rodríguez (2004) investigaram a melhoria nas propriedades de impacto das misturas de poliestireno/SBR produzidas por diferentes concentrações e tipos de estireno-butadieno (SBR). As amostras foram irradiadas por diferentes doses de radiação gama para atingir uma boa aderência e uma boa estabilidade, entre a fase de borracha e a matriz de poliestireno, para melhorias nas propriedades de impacto. Os resultados mostraram que a maior resistência ao impacto foi obtida para a mistura com $10 \%$ de SBR, 30\% de estireno, uma conformação linear, uma micro-estrutura de 53\% de trans, 38\% cis e $9 \%$ de vinil, uma massa molar de 207.000 e uma dose de $100 \mathrm{kGy}$. Várias amostras foram preparadas com $0 \%, 3 \%, 5 \%$ e $10 \%$ de SBR e caracterizadas por FTIR e espectroscopia Raman. Foi possível obter blendas com até $263 \%$ de melhoria nas propriedades de impacto em relação ao PS não modificado. Para amostras irradiadas, um possível mecanismo para explicar o aumento da tenacidade do material é a produção de ligações químicas cruzadas entre o anel benzeno do poliestireno e a matriz de polibutadieno.

Abreu, Forte e Libermam (2006) produziram 
blendas de polipropileno e elastômeros termoplásticos (TPEs), estireno-butadienoestireno (SBS) e estireno-b-etileno-co-butileno-bestireno (SEBS). Estas blendas foram preparadas com o objetivo de avaliar a influência do tipo e da concentração do elastômero nas propriedades mecânicas e na morfologia das blendas. Foram utilizados dois tipos de polipropileno, um homopolímero de propileno (PP-H) e um copolímero randômico de propileno-etileno (PP-R), sendo avaliado também o efeito das características da matriz termoplástica. O elastômero termoplástico aumentou a resistência ao impacto do PP, e a variação da rigidez das blendas foi dependente somente da quantidade de TPE adicionada, sendo estas comparativamente mais rígidas que aquelas com igual teor de elastômero convencional, tipo EPDM e EPR. A blenda com melhor balanço rigidez-impacto foi aquela de PP-R com 10\% de SEBS. As blendas do copolímero de propileno-etileno com os TPEs apresentaram maior deformação do que aquelas com o homopolímero, devido à natureza menos cristalina da matriz do copolímero de propileno. As blendas tanto do homo quanto do copolímero de propileno com SEBS ficaram mais homogêneas em função da maior afinidade do bloco central poliolefínico EB (etileno-co-butileno) do primeiro com a região amorfa da matriz, sendo esta mais significativa no PP-R.

Coutinho et al. (2007) avaliaram a tenacificação de poliestireno (PS) utilizando três tipos de polibutadieno $(\mathrm{PB})$ : polibutadieno baixo-cis $\left(\mathrm{PB}_{\mathrm{b}}\right)$, polibutadieno alto-cis $\left(\mathrm{PB}_{\mathrm{a}}\right)$ e copolímero em bloco (SBS) de estireno e butadieno $\left(\mathrm{PB}_{\mathrm{co}}\right)$. Observaram um aumento na resistência ao impacto de 138, 208 e $823 \%$, quando utilizados polibutadieno baixo-cis, polibutadieno alto-cis e copolímero em bloco de estireno e butadieno, respectivamente. As condições de processamento tiveram influência na qualidade dos materiais obtidos e temperaturas de processamento superiores a $180^{\circ} \mathrm{C}$ provocaram a degradação do material. As misturas PS/PB exibiram morfologia bifásica com domínios de polibutadieno dispersos aleatoriamente na matriz do PS, com diâmetro de partícula inferior a $1 \mu \mathrm{m}$. Essas misturas apresentaram baixa adesão entre as fases termoplástica e elastomérica e uma alta tensão interfacial. A incorporação de material elastomérico à matriz do PS produziu materiais opacos com alta resistência ao impacto. As misturas $\mathrm{PS} / \mathrm{PBa}$ apresentaram valores de resistência ao impacto superiores aos das misturas $\mathrm{PS} / \mathrm{PB}_{\mathrm{b}}$ e muito inferiores aos das misturas $\mathrm{PS} / \mathrm{PB}_{\mathrm{co}}$. Observaram que todos os materiais tiveram resistência ao impacto superior a do HIPS comercial e apresentaram comportamento pseudoplástico, viscoelasticidade e diferentes energias de ativação para o escoamento sob as condições de cisalhamento estudadas.

Ribeiro, Domingues Jr e Riegel (2012) avaliaram a recuperação das propriedades de poliestireno de alto impacto (HIPS) reciclado através da incorporação de copolímeros estirenobutadieno-estireno (SBS) de estrutura linear e radial. As blendas foram processadas em uma extrusora dupla rosca. Os copolímeros foram adicionados em teores de 5, 10 e 15\% em massa. As propriedades avaliadas foram o índice de fluidez, a morfologia e o comportamento frente a ensaios de tração, flexão e resistência ao impacto das blendas formuladas. Os valores de resistência ao impacto das misturas HIPS reciclado/SBS radial foram maiores que os encontrados para as misturas com SBS linear. A maior recuperação de propriedades de impacto foi verificada com a adição de $15 \%$ de SBS radial. Neste teor, verificou-se que a resistência ao impacto do HIPS reciclado aumenta em três vezes, aproximadamente (de 24,6 J.m $\mathrm{m}^{-1}$ para 74,7 J). Contudo, a adição de 15\% de SBS linear também apresentou aumento versus o HIPS não modificado (de 24,6 J.m ${ }^{-1}$ para 57,0 J). Os ensaios de tração e flexão indicaram uma diminuição da rigidez de todas as composições com o aumento do teor de copolímero SBS observado pela diminuição do módulo elástico e aumento da deformação na 
ruptura.As micrografias das composições HIPS/SBS sugerem que as partículas de copolímero SBS foram arrancadas da matriz de HIPS reciclado, formando vazios com rompimento da interface. O tamanho maior dos vazios nas composições com SBS radial provavelmente está associado ao tamanho das partículas dispersas, efeitos de cavitação favorecidos pela estrutura tipo estrela e à maior massa molecular deste copolímero, o que, novamente, concorda com os valores de maior resistência ao impacto obtido em todas as composições de SBS radial quando comparados aos das composições com iguais teores de SBS linear.

Zhang et al. (2013) prepararam blendas de resíduos de pó de borracha/poliestireno (WRP/PS) em diferentes proporções em peso com poliestireno enxertado com copolímero de borracha de estirenobutadieno (PS-g-SBR) como compatibilizante. O PSg-SBR foi sintetizado pelo método de polimerização em emulsão e confirmado por meio de espectroscopia de infravermelho com transformada de Fourier (FTIR), calorimetria diferencial de varredura (DSC). O copolímero em diferentes proporções em peso foi posteriormente adicionado às misturas. Os efeitos da razão de peso de WRP/PS e compatibilizante nas propriedades mecânicas foram investigados. A mistura PS/WRP numa proporção em peso de 80/20 mostrou resistência ao impacto alta. E a resistência ao impacto foi ainda mais elevada com a adição de SBR-g-PS. Entretanto, a resistência ao impacto foi bastante reduzida com um alto teor de copolímero. A morfologia e propriedades térmicas das misturas WRP/PS foram examinadas por DSC, microscopia eletrônica de varredura (MEV) e termogravimetria (TG). Os resultados de DSC indicaram que, em comparação com a mistura de PS/WRP, a temperatura de transição vítrea $(\mathrm{Tg})$ do poliestireno na mistura PS/WRP/SBR-g-PS foi deslocada para valores mais baixos de temperatura por causa da formação de ligações químicas cruzadas entre PS e WRP e, a Tg da fase WRP de ambas as misturas PS/WRP e PS/ WRP/SBR-g-PS não apareceu. Os resultados de MEV indicaram que a adesão interfacial nas misturas com o copolímero PS-g-SBR foi melhorada. A morfologia apresentou uma estrutura com o PS e WRP apresentando fase contínua e fase descontínua, respectivamente, indicando a adesão interfacial moderada entre WRP e a matriz PS.

Yousefi(2013)estudoublendas depoliestireno(PS) e estireno-butadieno (SBR) misturadas fisicamente no estado fundido em diferentes proporções para formar misturas físicas de elastômeros termoplásticos. Esta mistura polimérica deverá comportar-se mais ou menos semelhante aos copolímeros em bloco sintetizados quimicamente, tais como copolímeros em bloco de estireno-butadieno-estireno(SBS). Neste estudo, as propriedades mecânicas e termomecânicas foram investigadas e comparadas as do SBS. A mistura apresenta uma morfologia de duas fases com uma interface, que mostra interações muito fracas. De acordo com a morfologia e o tamanho do domínio da fase dispersa a mistura apresenta compatibilidade. As propriedades mecânicas das misturas confirmaram a compatibilidade e a eficácia da transferência de tensão na interface. As propriedades de impacto e tração foram melhoradas com o aumento do teor de SBR na mistura.

\section{Conclusão}

O desenvolvimento de blendas poliméricas é uma abordagem simples e econômica das modificações das propriedades dos polímeros, resultando num novo material com propriedades específicas. Umadas principais técnicas utilizadas pelas industriais para tenacificação dos polímeros é a misturas de polímeros, no qual uma das fases é a borracha. Os rejeitos de borrachas advindos das indústrias de pneus e calçados podem ser reaproveitados no processo de tenacificação de polímeros, bem como minimizar o efeito nocivo deles sobre o meio ambiente $\mathrm{e}$ agregar valor a um material que seria descartado.

A mistura de polímeros é uma rota economicamente viável para melhorar as propriedades dos materiais poliméricos já existentes obtendo materiais com 
características físicas, químicas e físico-químicas diferenciadas. Os componentes da blenda são selecionados de modo a conservar as vantagens de cada polímero.

A grande atenção dada às blendas poliméricas, tanto do ponto de vista acadêmico, quanto industrial/comercial, é devido à relativa facilidade na obtenção de materiais com propriedades desejadas, sem altos investimentos aplicados no desenvolvimento da síntese de novos polímeros. Dessa maneira, as blendas poliméricas são uma versátil solução tecnológica para se obter materiais poliméricos com as mais diversas especificações a um custo relativamente baixo por meio de combinações de polímeros com as propriedades de interesse.

\section{Agradecimentos}

Os autores agradecem a UFCG, ao CNPq/PIBIC, ao $\mathrm{MCTI} / \mathrm{CNPq}$ e a CAPES pelo apoio financeiro.

\section{Referências}

ARAÚJO, S. S. Estudo das alternativas tecnológicas de reaproveitamento e reutilização de pneus provenientes de descarte. 2011. Trabalho de Conclusão de Curso (Graduação em Engenharia de Materiais e Metalúrgica) - Universidade Federal de Campina Grande, Campina Grande.

ARAÚJO, E. M.; CARVALHO, L. H.; FOOK, M. V. L. Propriedades mecânicas de Blendas Ps/ resíduos de borracha-influência da concentração, granulometria e método da moldagem. Polímeros: Ciência e Tecnologia, v.19, n.3, p. 45-52, 1997. DOI: $10.1590 / \mathrm{S} 0104-14281997000300008$

AMÂNCIO, D. A.; MEGOD, M. O. A. Avaliação das propriedades mecânicas de blendas de polietileno de baixa densidade (PEBD) com polivinil butiral (PVB) reciclado de vidro laminado automotivo. In: JORNADA DE INICIAÇÃO CIENTÍFICA, 7., 2011, São Paulo. Universidade Presbiteriana Mackenzie.
ARAÚJO, E. M. Tenacificação da poliamida $6 \mathrm{com}$ ABS por meio da técnica de compatibilização in situ com o uso de copolímeros acrílicos reativos. 2001. Tese (Doutorado em Ciência e Engenharia de Materiais) Programa de Pós-Graduação em Ciência e Engenharia de Materiais (PPG-CEM/UFSCar) - São Carlos.

ARAÚJO, E. M.; HAGE JR, E.; CARVALHO, A. J. F. Compatibilização de Blendas de Poliamida 6/ABS usando os Copolímeros Acrílicos Reativos MMAGMA e MMA-MA. Parte 1: comportamento reológico e propriedades mecânicas das blendas. polímeros. Ciência e Tecnologia, v. 13, n. 3, p. 205-211, 2003. DOI: $10.1590 / \mathrm{S} 0104-14282003000300011$

AJJ, A.; UTRACKI, L.A. Interphase and Compatibilization of Polymer Blends. Polymer Engineering \& Science, v. 36, n. 12, p. 1574-1585, 1996. DOI: $10.1002 /$ pen. 10554

ABREU, F. O. M. S.; FORTE, M. M.; LIBERMAN, S. A. Propriedades mecânicas e morfologia de blendas de polipropileno com TPEs. Polímeros: Ciência e Tecnologia, v. 16, n. 1, p. 71-78, 2006. DOI: 10.1590/ S0104-14282006000100015

ASSOCIAÇÃO NACIONAL DA INDÚSTRIA DE PNEUMÁTICO - ANIP. Volume de Produção de Pneus. Disponível em: < http:/www.anip.com.br/?cont=home >. Acesso em: 14 Março de 2015.

AKCELRUD, L. Fundamentos da ciência dos polimeros. Barueri: Manole, 2007.

BOM, R. P. Caracterização de resíduos plásticos procedentes da reciclagem de papel. Estudos Tecnológicos, v. 5, n. 2, p. 147-156, 2009. DOI: 10.4013/ ete. 2009.52 .03

CARMO, D. S.; MAIA, N. S.; CÉSAR, C. G. Evaluation of the typology of construction waste selivered to processing plants in Belo Horizonte. Revista de Engenharia Sanitária e Ambiental, v. 17, n.2, p. 187192, 2012. DOI: 10.1590/S1413-41522012000200008

COSTA, M. P. M. Estudo da tenacificação do poliestireno com elastômero butadiênicos. 2009. 92f. Dissertação (Mestrado em Química) - Centro de Ciências e Tecnologia, Universidade do Estado do Rio de Janeiro, Rio de Janeiro. 
COLLATTO, D.; BERGMANN, C. P.; RAMIRES, M. V. V. Influência da Granulometria do Resíduo de Celulose nas Propriedades do Material Cerâmico. Estudos Tecnológicos, v. 4, n. 1, p. 1-11, 2008.

COUTINHO, F. M. B.; COSTA, M. P. M.; GUIMARÃES, M. J. C.; SOARES, B. G. Estudo Comparativo de Diferentes Tipos de Polibutadieno na Tenacificação de Poliestireno. Polímeros: Ciência e Tecnologia, v. 17, n. 4, p. 318-324, 2007.

\section{DOI: $\quad$ DOI: 10.1590/S0104-14282007000400011}

CHEN, C.C.; WHITE, J.L. Compatibilizing Agents in Polymer Blends: Interfacial Tension, Phase Morphology and Mechanical Properties. Polymer Engineering and Science, v. 33, n. 14, p. 923-930, 1993. DOI: $10.1002 /$ pen.760331409

FERNANDES, L. L.; FREITAS, C. A.; DEMARQUETTE, N. R.; FECHINE, G. J. M. Estudo do Efeito do Tipo de Polipropileno na Fotodegradação da Blenda Polipropileno /Poliestireno de Alto Impacto. Polímeros: Ciência e Tecnologia, v. 22, n. 1, p. 61-68, 2012. DOI: 10.1590/S0104-14282012005 000013

GOMES FILHO, C. V. Levantamento do potencial de resíduos de borracha no Brasil e avaliação de sua utilização na indústria da construção civil. 2007. 138f. Dissertação (Programa de Pós-Graduação em Tecnologia) - Instituto de Engenharia do Paraná, Curitiba.

GAN, P. P.; PAUL, D. R. Phase Behavior of Blends of Styrene/Maleic Anhydride Copolymers. Journal of Applied Polymer Science, v. 54, n. 3, p. 317-331, 1994. DOI: 10.1002/app.1994.070540306

HAGE JR. E.; PESSAN, L. A. Aperfeiçoamento em tecnologia de plásticos. Módulo 7: blendas poliméricas. São Carlos: ABPol, 2001.

KONING, C.; DUIN, M. V.; PAGNOULLE, C.; JEROME, R. Strategies for compatibilization of polymer blends. Progress in Polymer Science, v. 23, n. 4, p. 707-757, 1998. DOI: 10.1016/S00796700(97)00054-3

MANO, E. B.; MENDES, L. C. Introdução a polímeros. 2. ed. São Paulo: Edgard Blüncher, 2001.
MARTÍNEZ-BARRERAG.;LÓPEZ,H.; CASTAÑO, V. M.; RODRÍGUEZ, R. Studies on the rubber phase stability in gamma irradiated polystyrene-SBR blends by using FTIR and Raman spectroscopy. Radiation Physics and Chemistry, v. 69, n. 2, p.155-162, 2004. DOI: 10.1016/S0969-806X(03)00452-3

MOTHE, C. G.; AZEVEDO, A. D. Análise térmica de materiais. São Paulo: Comercial, 2002.

OLIVEIRA, A. D. Dispersão seletiva de argila montmorilonita em blendas poliméricas de PA6/ ABS. 2009. 103f. Dissertação (Mestrado em Ciência e Engenharia de Materiais) - Programa de PósGraduação em Ciência e Engenharia de Materiais (PPG-CEM/UFSCar), São Carlos.

PASSADOR, F. R.; PESSAN, L. A.; RODOLFO JR, A. Estado de mistura e dispersão da fase borrachosa em blendas PVC/NBR. Polímeros: Ciência e Tecnologia, v. 16 , n. 3 , p. $174-181$, 2006. DOI: $10.1590 /$ S010414282006000300005

NEWMAN, S.; PAUL, D. R. Rubber modification of plastics. New York: Academic Press, 1978. v. 2.

LUNA, C. B. B.; SILVA, D. F.; ARAÚJO, E. M. Análise do comportamento termomecânico, térmico e mecânico de blendas de PA6/resíduos de borracha. Revista de Engenharia e Tecnologia, v. 6, n. 1, p. 160169, 2014.

RIBEIRO, V. F.; DOMINGUES JR, N. S. D.; RIEGEL I. C. Estudo da recuperação das propriedades de poliestireno de alto impacto (HIPS) através da incorporação de borracha termoplástica tipo estirenobutadieno-estireno (SBS). Polímeros: Ciência e Tecnologia, v. 22, n. 2, p. 186-192, 2012. DOI: 10.1590/S0104-14282012005000023

ROVERE, J.; CORREA, C. A.; GRASSI, V. G.; PIZZOL, M. F. D. Caracterização morfológica do poliestireno de alto impacto (HIPS). Polímeros: Ciência e Tecnologia, v. 18, n. 1, p. 12-19, 2008. DOI: 10.1590/S0104-14282008000100007

SILVA, D. F. Desenvolvimento de Blendas de Poliamida 6/Composto de Borracha Reciclada. 2011. 89f. Dissertação de Mestrado, Universidade Federal de Campina Grande, Campina Grande-PB. 
SILVA, D. F.; ARAÚJO, E. M.; MELO, T. J. A. Desenvolvimento de blendas de poliamida 6/composto de borracha reciclada (SBRr). Revista Eletrônica de Materiais e Processos, v. 7, n. 2, p.104-110, 2012.

SCHUSTER, R. H. Recycling of rubber: problems and strategies. In: CONGRESSO BRASILEIRO DE POLÍMEROS, 11., 1993, São Paulo. Anais... São Paulo, 1993. v. 2, p. 1135-1143.

STAMM, M.; SCHUBERT, D. W. Interface between incompatible polymers. Annual Review Materials Science, v. 25, n. 3, p. 325-356, 1995. DOI:10.1146/ annurev.ms.25.080195.001545

SPERLING, L. H. Polymeric multicomponent materials: an. introduction. New York: John Wiley \& Sons, 2001.

UTRACKI, L.A. Polymer Alloys and Blends: Thermodynamics and Rheology. Munich: Hanser Publishers Inc., 1989.

WIEBECK, H.; HARADA, J. Plásticos de engenharia: tecnologia e aplicações. São Paulo: Artliber, 2005.

YOUSEFI, A. A. Segmental mobility in the vicinity of $\mathrm{tg}$ in $\mathrm{ps} / \mathrm{sbr}$ blends: nanodomain size prediction of the dispersed phase. Journal of Applied Polymer Science, v. 127, n. 1, p. 659-665, 2013. DOI: 10.1002/ app. 37847

ZHANG, J.; CHEN, H.; ZHOU, Y.; KE, C.; LU, H. Compatibility of waste rubber powder/polystyrene blends by the addition of styrene grafted styrene butadiene rubber copolymer: effect on morphology and properties. Polymer Bulletin, v. 70, n. 10, p. 28292841, 2013. DOI: 10.1007/s00289-013-0991-3 
